\title{
DIAGNOSTIC IMPORTANCE OF COVID-19 PANDEMIC
}

\author{
Kafle $\mathrm{SU}^{*}$ \\ Associate Professor, Department of Pathology, Birat Medical College Teaching Hospital \\ https://orcid.org/0000-0002-7743-0485
}

\author{
Citation \\ Kafle SU, Diagnostic Importance of COVID-19 Pandemic, BJHS 2020, 5(1)11:1000
}

DOI: https://doi.org/10.3126/bjhs.v5i2.31370

In this ongoing COVID-19 pandemic session, there is seen lot of new questions and challenges regarding the tests for this novel Severe Acute Respiratory Syndrome Coronavirus 2 (SARS-CoV-2) increasingly available (though still limited and/or slow in some regions).

As SARS-CoV-2 is genuine newer virus, the diagnostic modality and test utilization for detecting such virus has little evidence on its well-fixed protocols and diagnostic stewardship. Whom to target first for test? When is the proper/exact timing to test? What exactly to test on that time? How often to test? Numerous varieties of such queries are always there to be answered; particularly, what types of tests are required and available under which circumstances are they useful? Such things if understood and work out for implementation will definitely help in guiding the importance of testing at the local, regional and national levels and inform those policy makers who assess the supply chain to ensure that needed testing is and continues to be available.

At this stage in the face of a rapidly changing and neverbefore-experienced situation, we explain here the types of diagnostic tests available, which might be useful. Moreover, our mind should not forget that we are experiencing: a new virus during an unpredicted pandemic in latest modern era. Keeping such things in mind, the diagnostic testing modalities has become an especially important weapon in the absence of either proven effective therapy or a vaccine. This in turn can lead to inform patient management and potentially serving to save lives by limiting the spread or dissemination of SARSCoV-2. Basically, the most important aspect in such context becomes important to determine who is currently infected and to determine who has previously been infected. In continuum, the rationale and purpose for such test is to conduct research and surveillance in virology, drug and vaccine development, clinical services, epidemiology and public health.

There exist basically two broad categories of tests that detect SARS-CoV-2: those that detect the virus itself and those that detect the host's response to the virus. RT-PCR tests can determine "currently infected person, provide a basis for isolation and infection prevention/control procedures, enable contact tracing and guide the clinical management of those

\section{REFERENCE}

1. Patel R, Fang FC. 2018. Diagnostic stewardship: opportunity for a laboratory-infectious diseases partnership. Clin Infect Dis 67:799 801. doi: $10.1093 /$ cid/ciy077 with symptoms suggestive of COVID-19". Likewise, antibody tests can determine "who has ever been infected and who may be immune to re-infection". This can be very helpful for guiding strategies for non-pharmaceutical interventions such as social distancing measures. Depending on the timing and accuracy of the test, they can potentially also identify who is currently infected. The evaluation of the accuracy of a test or diagnostic examination can be done incorporating the sensitivity and specificity indices of a test. The interpretation can be as: "those testing positive who have the disease are called "true positives"; those testing positive who do not have the disease are called "false positives"; those testing negative who have the disease are called "false negatives"; and those testing negative who do not have the disease are called "true negatives".

Regarding the other diagnostic options, viral culture and isolation can be an ideal way. Although the virus can be cultured, this is dangerous and not routinely done in clinical laboratories. While detection of viral antigens is theoretically possible, this approach has not been a primary one till date, but can be considered for further research. Radiological imaging: Chest $X$ ray but especially Chest CT scan may be helpful in making the diagnosis, but no finding can completely rule in or rule out the possibility of COVID-19. Classic signs of Corona Virus-induced Pneumonia are described as 'ground glass' opacification. Other testing approaches that are in the pipeline are also being considered and reviewed by scientists that can identify segments of the virus. If the entire world's population could be tested all at once hypothetically, with a test providing $100 \%$ specificity and sensitivity (unrealistic, obviously), we might be able to identify all infected individuals and sort people into those who at that moment in time were asymptomatic, minimally/moderately symptomatic and severely symptomatic. Thus, then would become easier for sure to separate and move ahead treating them accordingly.

Considering this being an ideal situation as a guide, it is wise to accept as we do have available tests today should be carefully considered. Moreover, especially in the absence of therapeutics or vaccines adapting and implementing such tests can be useful in tackling up the current crisis closer to the ideal situation.

2. http://documents.worldbank.org/curated/en/14516158653 6712080/ Purpose-and-Options-for-Testing-for-SARS-Cov2-the-COVID-19-VirusConsiderations-for-World-Bank-Task-Teams-Managing-COVID-19-FastTrack-Facility Operations.

3. https://www.medscape.com/viewarticle/928150 